\title{
A Novel Interference Suppression Algorithm Based on Irregular Wavelet Packet Transform in DSSS Satellite Communication System
}

\author{
Yanghui Tong, Fangjun Liu, Daoxing Guo and Heng Wang \\ PLA University of Science and Technology, Nanjing, Jiangsu, China \\ email:1024276863@qq.com
}

\begin{abstract}
Keywords: satellite communications, DSSS, wavelet packet transform, Optimum wavelet base, interference suppression

Abstract: In recent year, the Direct Sequence Spread Spectrum (DSSS) technology has been introduced as an attractive approach to guarantee anti-interference capability in satellite communication system. However, due to the limitation of the DSSS, the system performance has been greatly deteriorated while a strong interference is out of the tolerance of the system. Meanwhile, what the wavelet packet transforms is ideally suitable for the interference detection and suppression of DSSS satellite communication system by exploiting its excellent local time-frequency domain analysis capability. In this paper, a novel algorithm has been put forward to achieving position and interference suppression through combing the sub-band power ratio with minimum power thresholds which are based on the analysis on the optimal wavelet packet decomposition. Simulation result shows that the proposed interference suppression algorithm significantly improves the anti-jamming capability of DSSS satellite communication system compared with the traditional FFT algorithm.
\end{abstract}

\section{Introduction}

With the advent of the information age, the requirements of accessing to information are increasingly urgent. Due to the anti-interference capacities of direct sequence spread spectrum (DSSS) systems, it has been widely used in both the military and civil communications. However, the anti-interference capability of DSSS system is limited by the processing gain. The performance of the system largely degrades when the interference power is greater than the jamming margin. It is of great significant to employ signal processing techniques to improve the anti-jamming performance of DSSS system.

Currently, the signal processing techniques is used in the spread spectrum system which is include time domain processing techniques [1,2] and transform domain processing structures [3, 4]. Time domain processing techniques can eliminate the narrowband interference (NBI) completely because it estimates the interference exactly and extract from the receive signal to leave the interference free DSSS signal. But it is necessary for convergence time to reach the optimal solution. So it is suitable for slow-altered interference. On the other hand, the changing interference can be quickly tracked by the transform domain suppression. The transform domain processing structures adopt the methods like the Fourier transform, wavelet transform and wavelet packet transform, and to convert a time-domain signal into the transform domain. It utilizes the different features between the interference signal and the desired signal in the frequency domain to make a distinction. It uses the relevant suppression method to eliminate the interference signals. Therefore, the transform domain processing techniques are able to track and capture dynamic interference quickly and adaptively.

Wavelet packet transform is very suitable for interference detection and suppression in the direct sequence spread spectrum systems because of its excellent local time and frequency domain analysis capabilities. The difficulty of using wavelet packet to achieve interference suppression is mainly reflected in two aspects: The first one is about how to carry out wavelet packet decomposition on the received signal. The second one is about how to locate interference on the basis of wavelet tree and to choose appropriate algorithms for achieving interference suppression.

As is known to all, the wavelet packet decomposition usually includes regular decomposition 
with uniform sub-band and optimal wavelet packet decomposition with uneven sub-band [5,6]. The common interference locating methods based on wavelet packet include sub-band power ratio node positioning [7], adaptive threshold positioning [8] and so on. A new method for interference suppression has been put forward based on the optimum wavelet packet decomposition. Firstly, locate the interference by using sub-band power ratio so that sub-band high-power interference can be suppressed. Then, make further judgments and suppression on the non-suppressed sub-band by using the minimum power threshold method in order to have a better eliminate residual interference.

\section{System model}

The interference suppression and noise suppression model, which is built based on wavelet packet, which is shown in Fig. 1. The signal from the ground receiver usually includes spread spectrum signal component, narrowband interference and noise components. The received signal $r(k)$ can be expressed as:

$$
r(k)=s(k)+J(k)+n(k)
$$

In this formula, the $s(k)$ denotes spread spectrum communication signal using the BPSK modulation, $J(k)$ presents the narrow-band interference signals, $n(k)$ denotes the additive white Gaussian noise signal with a mean zero and the variance $\sigma^{2}$. Thus, the signal $s(k)$ based on direct sequence spread spectrum system can be expressed as:

$$
s(k)=\sqrt{P} s_{0}(k) P N(k) \cos \left[\omega_{0} k+\phi\right]
$$

In this equation above, $P$ represents power level of the spread spectrum signal, $s_{0}(k)$ is the binary information bits, $P N(k)$ is the spreading sequence, $\omega_{0}$ is carrier frequency, $\phi$ is the phase.

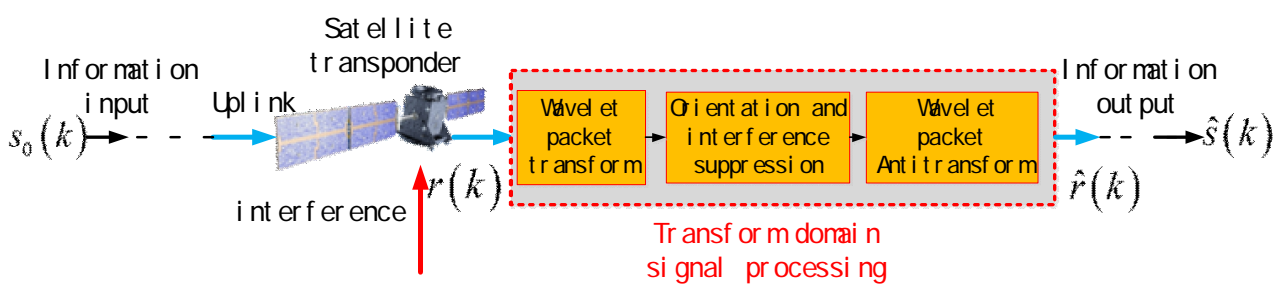

Fig.1 Schematic of DSSS interference suppression satellite communication based on wavelet packet transform

\section{Wavelet packet analysis basis of signal}

Wavelet packet transform has an excellent time-frequency localization features and multi-resolution analysis ability. When the interference changes in real-time, the interference will be located quickly and efficiently in a limited sub-band and then it will be eliminated through the relevant suppression algorithm.

The transform process of Wavelet packet is defined using the following sequence of functions with recursion:

$$
U_{2 n}(t)=\sqrt{2} \sum_{k \in Z} h(k) U_{n}(2 t-k) \quad U_{2 n+1}(t)=\sqrt{2} \sum_{k \in Z} g(k) U_{n}(2 t-k)
$$

In this formula, $U_{0}(t)$ is the scaling function of $\phi(t), U_{1}(t)$ is the mother wavelet of $\psi(t),\left\{U_{n}(t), n \in Z\right\}$ is known as Wavelet Packet Group of $U_{0}(t),\{h(k), k \in Z\}$ and 
$\left\{g(k)=(-1)^{-k} h(L-k-1), k \in Z\right\}$ respectively represents a low-pass filter coefficient group and high-pass filter set of coefficients of quadrature mirror filters QMF with supporting length L, and satisfy the following condition:

$$
\left\{\begin{array}{l}
\sum_{k \in Z} h(k-2 a) h(k-2 b)=\delta_{a, b} \\
\sum_{k \in Z} h(k)=\sqrt{2}
\end{array}\right.
$$

Wavelet packet needs to be discreted for practical applications. A following recursive discrete wavelet packet transform was given by C.K.Chui[9] :

$$
\left\{\begin{array}{l}
S_{l+1}^{2 n}(i)=\sum_{k \in z} h(i-2 k) \times S_{l}^{n}(k) \\
S_{l+1}^{2 n+1}(i)=\sum_{k \in z} g(i-2 k) \times S_{l}^{n}(k)
\end{array}\right.
$$

The corresponding inverse discrete wavelet packet transform is as follows:

$$
S_{l}^{2}(i)=\sum_{k \in z} h(i-2 k) S_{l+1}^{2 n}(k)+\sum_{k \in z} g(i-2 k) S_{l+1}^{2 n}(k)
$$

In this formula, $l$ represents the corresponding layers of wavelet packet decomposition, $n$ indicates the lateral nodes position of the corresponding level; $S_{l}^{n}$ represents the decomposition sequence of node $n$ at the layer of $l$. The received signal can be separated to a uniform or non-uniform spectral sub-band by making use of wavelet packet transform.

\section{Wavelet packet decomposition of the DS signal}

The optimum wavelet packet base is found out by the rule of energy compact in this paper, the specific steps are as follows:

(1) Make use of $M$-ary wavelet packet to decompose the received spread spectrum signal to gain a $M$-ary rules wavelet packet decomposition tree. The number of nodes contained in every layer is $M^{(L-1)}$.

(2) Each node uses a standard energy compact $G$ to analyze:

$$
G=\sigma^{2} /\left[\prod_{k=1}^{M} \sigma_{k}^{2}\right]^{\frac{1}{M}}
$$

$\sigma^{2}$ is the coefficient variance of node $N$ of layer $i(1 \leq i \leq L), \sigma_{k}^{2}$ is the coefficient variance of the child node $N_{k}$ whose number is $M$ and parent node is $N$ of the layer $i+1$. The threshold is $T h_{-} E C M$, if the standard $G$ satisfies the condition $G>T h_{-} E C M$, it indicates that the energy of child node $N_{k}$ is uneven so it need keep on decomposing; if the standard $G$ satisfies the condition $G \leq T h_{-} E C M$, it indicates that the energy of child node $N_{k}$ is even and the most parts of its frequency band are composed of spread spectrum signal element or interference element, so in this case the node will no longer make further decomposition. It is worth noticing that it needs to make interference judgment of the leaf nodes that are out of breaking down at the time of interference position and suppression to avoid the influence of interference components on system performance.

(3) If the node needs further decomposition, doing wavelet packet transform again is necessary to have the child node $N_{k}$ of the layer $i+2$, and then repeat step (1) in a similar operation until the 
decomposition achieves the given maximum number of scale $L$ (At this time it corresponds to the maximum frequency resolution ), the nodes stop decomposing, and we will get an optimum wavelet packet base, the diagram shown in Fig. 2 is an excellent example.

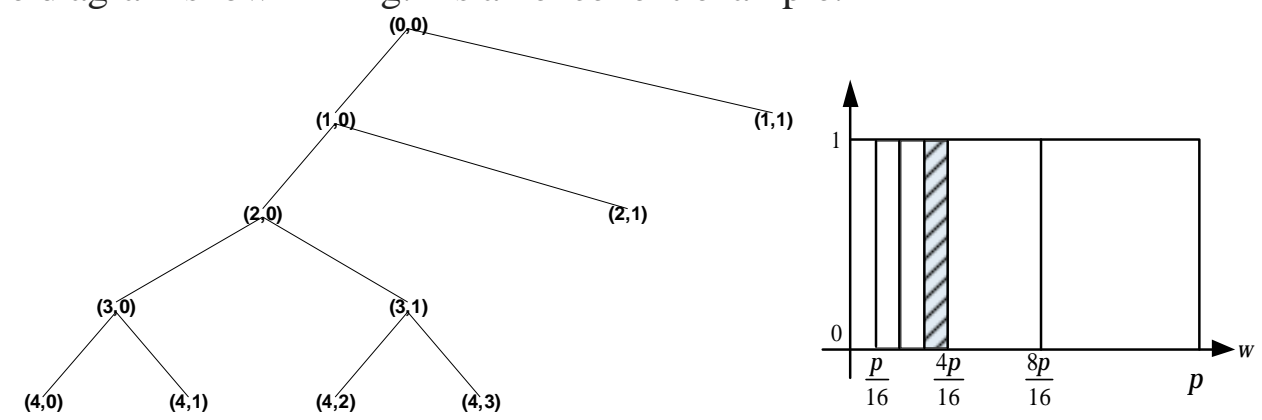

Fig.2 Diagram of optimal wavelet packet decomposition tree (decomposition level of 4) and the corresponding range bandwidth decomposition

\section{The interference positioning and suppression algorithm on the basis of wavelet packet transform.}

The steps present the interference suppression method of combining the sub-band power ratio with the minimum threshold in this article are as follows:

(1) Read the leaf node leaves of optimal wavelet packet tree, look for double leaves nodes which have the same parent node, and process those double leaves nodes with the sub-band power ratio interference positioning suppression. Then use the minimum threshold to judge the existence of the residual interference, if there is interference, then the coefficient of this leave is set as zero directly;

(2) Search for single leaf nodes which have different parent nodes. According to the process of optimal wavelet packet decomposition, there may be interference left on the decomposed single leaf nodes. In order to achieve better inhibitory effect, read coefficients of all leaf nodes and calculate the variance firstly, then make use of the median in the variance collection to judge those leaves. If the variance of the leaves is greater than the median, it indicates that interference exists in the single leaf node, then the coefficient of this leave should be set as zero directly, otherwise, there is no interference in this single leaf node, which avoids the influence when the single leaf node is affected by interference. At the same time, it also ensures that most of the useful signals are undamaged;

(3) Transform the processed wavelet packet tree inversely by adopting M-ary wavelet packet and obtain the spread spectrum signal through interference suppression, then make despreading, demodulation and other subsequent processing.

\section{The simulation results and analysis}

In the simulation process, the DS system uses a pseudo-random sequence of length 32 to spread, modulation scheme is BPSK modulation, wavelet packet decomposition takes generation function of db16 (Daubechies wavelets), M of M-Ary takes two, the channel of AWGN is taken. Because the 
spreading code length is 32 , the system has a certain anti-interference tolerance, in order to reflect the performance of the algorithm for interference suppression well, jamming-to-signal ratio (JSR) must be greater than $15.051 \mathrm{~dB}\left(10 \times \log _{10}(32)=15.051 \mathrm{~dB}\right)$, jamming-to-signal ratio is from 20 to $50 \mathrm{~dB}$ in simulation process. The maximum decomposition level of wavelet packet tree is 5 , what is needed to satisfy both the suppression performance and the complex of decomposition. The normalized digital frequencies of two-tone interference to spreading rate are 0.314 and 1.57 , phase in $[0,2 \pi]$ is uniform.

From the Fig.3, it can be seen that the interference for the DS system poses a serious deterioration, it can not communicate properly without suppression, while the transform domain processing structures can effectively improve the performance of the system. Furthermore, the novel interference suppression algorithm based on irregular wavelet packet transform of combining the sub-band power ratio and the minimum threshold value has the better inhibitory effect than the traditional FFT interference suppression.

From the Fig.4, in the same condition that the signal-to-noise ratio ( $\mathrm{SNR}$ ) is $8 \mathrm{~dB}$, when $\mathrm{SNR}$ is low, the performance of the novel interference suppression algorithm based on irregular wavelet packet transform of combining the sub-band power ratio and the minimum threshold value is close with the traditional FFT interference suppression. But as the SNR increases, the anti-interference effect of the latter is significantly better than the former.

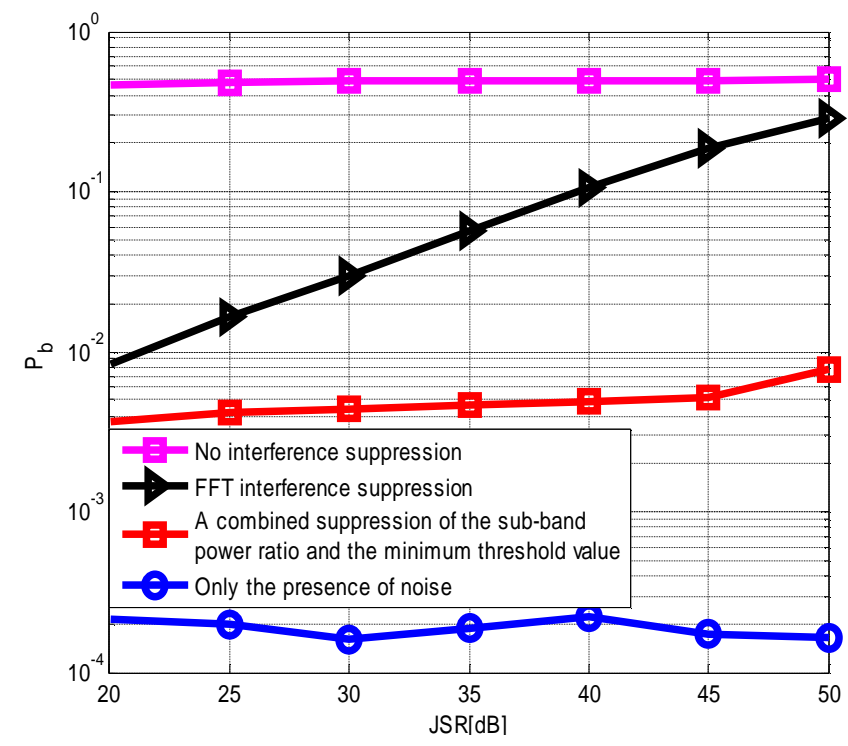

Fig.3 The BER performance comparison of various algorithms in different jamming-tosignal ratio and two-tone interference

$$
(\mathrm{SNR}=8 \mathrm{~dB})
$$

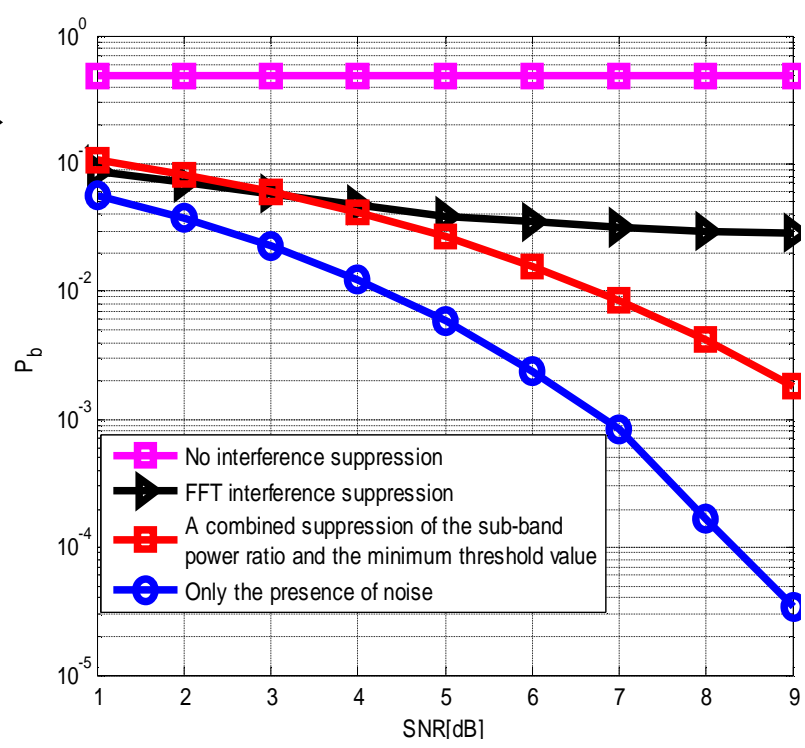

Fig.4 The BER performance comparison of various algorithms in different signal-tonoise ratio and two-tone interference

$$
(\mathrm{JSR}=30 \mathrm{~dB})
$$

\section{Conclusion}

A novel interference suppression algorithm that irregular wavelet packets transform by combining the sub-band power ratio and the minimum threshold is proposed in this paper. Our 
proposed algorithm limits the interference to a few number of sub-bands to avoid damage to the useful signal while thoroughly eliminates the residual interference with the assistance of sub-band power ratio suppression. Simulation results reveals that interference are effectively located and suppressed even through under the scenario of strong interference, which significantly enhance the anti-interference ability of DSSS satellite communication system.

\section{References}

[1]. K.C.Ho, Xiaoning Lu, Vandana Metha. Adaptive Blind Narrowband Interference Cancellation for Multi-User Detection[J]. IEEE Transactions on Wireless Communications, 2007,6(3):1024-1033.

[2]. Rodrigo C.de Lamare, Martin Haardt, Raimundo Sampaio-Neto. Blind Adaptive Constrained Reduced-Rank Parameter Estimation Based on Constant Modulus Design for CDMA Interference Suppression[J]. IEEE Trans on Signal Processing, 2008, 56(6): 2470-2482.

[3]. Chunhai Zhang, Lijun Xue, Eryang Zhang. Narrow-band interference suppression in transform domain based on adaptive multi-threshold algorithm [J]. Journal of Electronics \& Information Technology, 2006, 28(3):461-465.

[4]. P.Azmi and M.Nasiri-Kenari. Generalised Fourier transform-domain technique for narrowband interference suppression in CDMA communication system[J]. Electronics Letters, 2001,37(10):652-654.

[5]. Shi Xianjun, Lin Sa, Li Ruiliang. Signal denoising algorithm and its application based on best wavelet packet basis[J]. Journal of Naval Aeronautical Engineering Institute, 2006,21(5):506-509.

[6].Jiang Yu, Xiao Hong, Teng Wei, Liu Xingpeng, Gao Hongyou, Yu Shaopeng. Wavelet-packet-transform for narrow-brand-interference suppression in direct sequence spread spectrum communication system[J]. Journal of Harbin University of Commerce,2008,24(1):98-100.

[7].Zhu Liwei, Jiang Pinqun. Adaptive Threshold Based on Wavelet Packet Transform for Narrowband Interference Suppression[J]. Journal of Data Acquisition and Processing,2013,28(6):843-847.

[8].Emilia Pardo, Miguel A. Rodriguez-Hernandez, Juan J.Perez-Solano. Narrowband interference suppression using undecimated wavelet packets in direct-sequence spread-spectrum receivers[J]. IEEE Transactions on Signal Processing, 2006,54(9):3648-3653.

[9]. Weimin Yang, Guangguo Bi. Rejection of narrowband interference in DSSS systems based on adaptive wavelet packet transform[J]. Journal of China Institute of Communications, 1999, 20(7):69-75. 